\title{
AUTOMATIC SPINAL FRACTURE DIAGNOSIS AND SURGICAL MANAGEMENT BASED ON 3D IMAGE ANALYSIS AND RECONSTRUCTION OF CT TRANSVERSE SECTIONS
}

\author{
Ming-Shium Hsieh ${ }^{1}$, Ming-Dar TSai ${ }^{2}$, Yi-Der YeH ${ }^{2}$, Shyan-Bin Jou ${ }^{3}$ \\ ${ }^{1}$ Department of Orthopaedics and Traumatology, Taipei Medical University Hospital, \\ Taipei Medical University, Taipei \\ ${ }^{2}$ Institute of Information and Computer Engineering, Chung Yuan Christian University, Chung-Li \\ ${ }^{3}$ Department of Information Management, Nanya Institute of Technology, Chung-Li, Taiwan
}

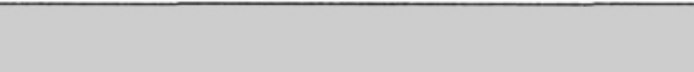

ABSTRACT

This paper describes an image analysis method that uses automatic algorithms for the evaluation of $3 D$ geometry of vertebral bones and spinal anatomic curve in the diagnosis of compression and burst fractures. The method uses a radial B-spline curve to approximate the ellipse-like vertebral body on a transverse section with a concave feature to evaluate the compression of the canal, and infers the anatomic curve of a vertebral body by linearly regressing the centers of $B$-spline approximate ellipse-like boundaries of the transversal sections passing the vertebral body. This method, then, calculates the reduced angle and height for recovering the compression fracture by comparing the regressed centerlines of neighboring bodies of the fracture body with the normal spinal anatomic curve. The prototype system can be used as a qualitative and quantitative tool for the diagnosis of compression and burst fractures using transverse sections, and for the instruction to plan accurate surgical procedures. An example demonstrates the fractured spine can be accurately diagnosed and instructed to operate by our method that achieved anatomic stability, released the syndrome of nerve compression and bone pain.
\end{abstract}

Biomed Eng Appl Basis Comm, 2002 (October); 14: 204-214.

Keywords: Image analysis, feature recognition, B-spline radial approximation, 3D image reconstruction, compression fracture, burst fracture, anatomic reduction and fusion.

\section{INTRODUCTION}

Patients with compression and burst fractures are not accurately diagnosed on the basis of either clinical findings (i.e., history taking and physical examination) or common imaging modalities including $x$-ray films,

Received: August 25, 2002; Accepted: Oct. 15, 2002 Correspondence:Ming-Dar Tsai, Ph. D., Professor Institute of Information and Computer Engineering, Chung Yuan Christian University

Chung Li, 32023 Taiwan

E-mail: tsai@ ice.cycu.edu.tw computed tomography (CT), CT combined with myelography and magnetic resonance imaging (MRI), etc [1-5]. Such problems result from the exact diagnosis of a spinal fracture must analyze the 3D geometry of compressed vertebral bones and burst bone fragments and the relations between the compressed bones with the spinal cord and their neighboring vertebral bones and disc herniation [6-9].

The diagnosis of a vertebral fracture must determine the classification and the degree of the fracture. A vertebral bone has three parts (posterior, middle and the anterior columns) that determine the classifications (compression, burst, seat-belt and shear dislocation) of the vertebral fracture [10-13]. Two kinds of compressed degrees must be evaluated: the deformation of 
anatomic curve and the reduction of the canal. These degrees determine the conservative or surgical modalities and procedures for the correction, reduction or decompression of the compressed bones and the spinal cord $[3,14-20]$. For example, the frequently applied instrumentation with posterior lateral pedicle screw (either for compression or distraction purposes) must use the information of deformed curve and reduction area of the canal to decide distracted disc and vertebral distances, and recovery spinal angles [3,20-23].

This paper proposes a method to determine the degree and classification of a vertebral fracture by analyzing the shape feature of the compressed bone and its neighboring bones, thus then determine a conservative or surgical modality and precise surgical procedures for the vertebral fracture. In this preliminary study, we evaluated the usefulness of our method by analyzing CT transverse sections of a fractured patient. The results were compared with data obtained by traditional clinical investigations, diagnosis, and operative findings.

\section{SUBJECTS AND METHODS}

\subsection{Theory Study}

Fig. 1(A) shows a fractured vertebral body in which the heights of anterior and middle columns are distinct to form an axis deformation of the vertebral bones (as shown in Fig. 1(B)). The fractured vertebral body may be sheared to form axis deviation. Some fragments of the fractured vertebral body may burst into the canal to reduce its diameter (Fig. 1(A)). The boundary of a vertebral body on a transverse (even litthe oblique) section can be considered as ellipse-like with a concave feature that forms the canal. If the transverse section (as Section $A$ in Fig. 1(B)) passes the upper of the vertebral body, the concave feature of the vertebral body and the bilateral pedicle and lamina

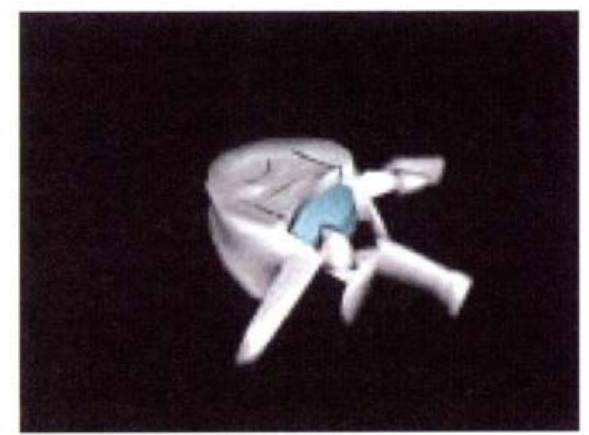

form a closed bone boundary at the canal. However, the boundary is not closed if the transverse section does not pass the pedicle and lamina (as Section $B$ in Fig. 1(B)).

A vertebral body on a transverse section can be approximated by a B-spline curve that has good approximation for circle, arc, sine or cosine- like boundaries $[24,25]$. For natural boundary, such as an ellipselike intervertebral disc boundary can be also well approximated as a B-spline radial and closed curve associated with concave and convex features. The convex features are then matched into a disc herniation feature then used to diagnose HIVD [26]. This study also approximates the boundary of a vertebral body on a transverse section as a B-spline radial and closed curve associated with a concave feature enclosing the canal.

The canal has the smallest diameter along the anteroposterior direction. Moreover, a canal has a large probability invaded by burst fragments of a vertebral body and pedicle during the vertebral fracture that mainly reduces the diameter along the anteroposterior direction. Therefore, a canal reduction during the vertebral fracture can be evaluated by the reduction of the diameter along the anteroposterior direction. Because transverse sections pass the fractured vertebral bone and its neighbors are performed as a routine, the compressed diameters of the canal on the sections of the fractured vertebral bone and the normal diameters on the sections of its neighbors can be compared to decide the compressed ratio of the canal.

Multiple transverse sections may pass the same vertebral bone to obtain different compressed ratio. The severest one is used to determine the degree of the fracture. The multiple sections also determine a centerline of the vertebral body. The angle between the centerlines of the above and below neighboring vertebral bodies to the fractured body can be used to compare with the normal anatomic angle between the center axis of the above and below neighboring vertebral

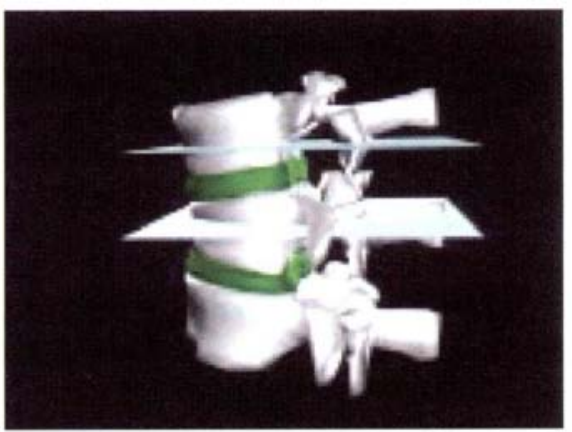

Fig.1 Spatial relation of a fractured vertebral bone with neighboring normal vertebral bodies $\triangle$ Gray area: bones. Green areas: disc spaces. Blue areas: bone fragment bursting to the canal. Black areas: cracks inside the body.

(A)A fractured vertebral bone in which the canal has a burst fragment at the canal and the body has cracks. (B)A spine with a fractured bone. Transverse section $A$ passes through the pedicle and lamina, while $B$ does not. 

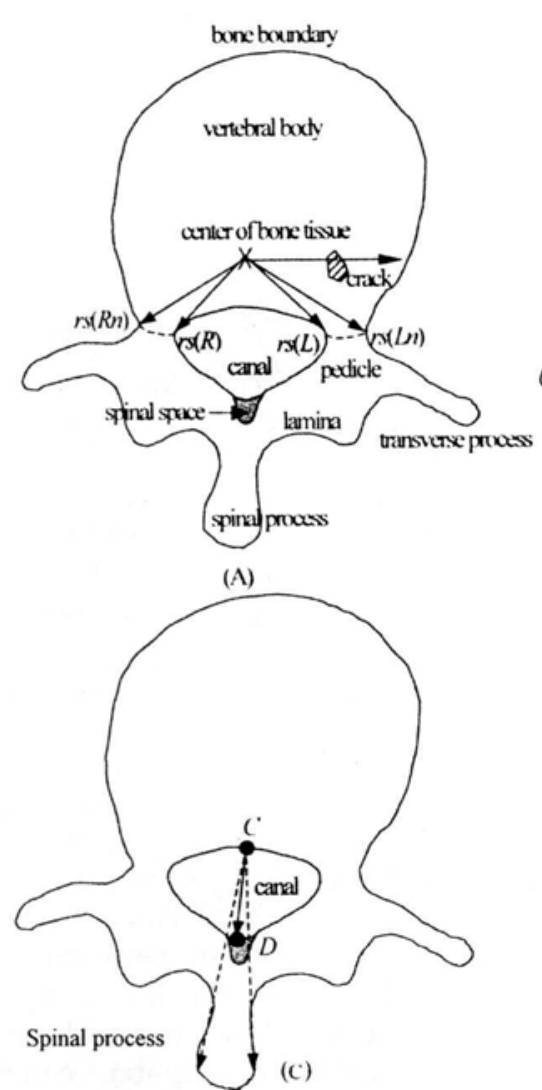

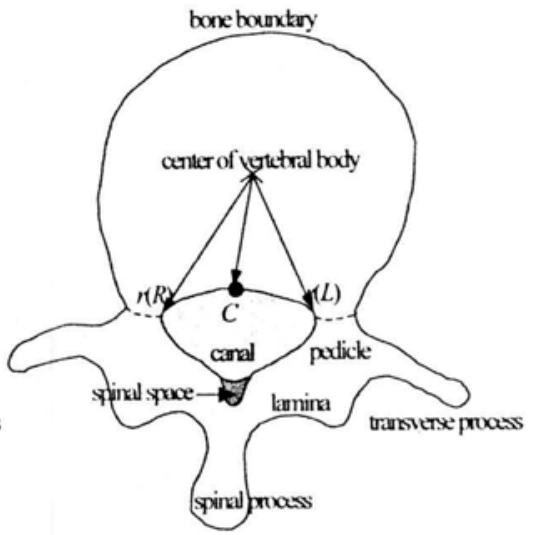

(B)

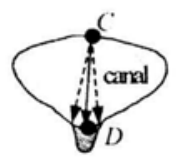

(D)

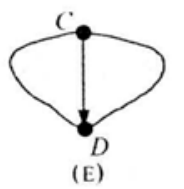

Fig.2 Image matching of bone boundary on a transverse section to obtain a B-spline approximate vertebral body.

(A)Determination of center of vertebral bone by averaging pixels of bone and disc substances. Determination of concave feature of the body enclosing the canal forming the canal by intersection computation.

(B)Determination of center and boundary of vertebral body by B-spline approximation

(C)Canal diameter determination by intersections of the vectors from the canal to spinal process

(D)Canal diameter determination by intersections of the vectors from the canal to spinal space

(E)Canal diameter determination by quadric approximation of the canal

bodies to determine the angle reduction for recovering the angle deformation. This heights of centerlines of neighboring bodies are used to determine a normal height of the fractured body to compare its fractured height, thus to determine the distracted or compressed distance.

\subsection{Vertebral Body Center Determination and Canal Diameter Calculation on a Transverse Section}

The process of matching a vertebral body boundary and canal diameter along the anteroposterior axis on a transverse section is described as follows.

1. Determine the center of bone tissues on a transverse section by averaging the position of the pixels of bone tissues (Fig. 2(A)). Because the area of the vertebral body on a transverse section is larger than the sum of pedicle and lamina, the center is located inside the vertebral body. A transverse section may pass a disc space in which disc substances and bone tissues coexist inside the ellipse-like boundary. For such transverse section, the pixels of disc substances are also used together with the ones of bone tissues to determine the center.

2. Use a vector starting from the center along every (totally 360 ) integral angular position to intersect the bone boundary. If multiple intersections were obtained, cracks or a hole exist inside the bone tissues. If the distance of two consecutive intersections of the same angular position is small, it is a crack that appears after the fracture and usually occurs at the vertebral body and pedicle [13]. If the distance of two consecutive intersections is large and a number of neighboring angular positions 
have such pairs of intersections, it is the canal boundary. When calculating the center of vertebral body, the crack intersections are ignored. From the center to lamina, pedicle and transverse process, multiple intersections (except the ones intersecting the cracks) are obtained. The left pedicle, lamina and transverse process can be excluded from the body using the boundary interpolated from the radius $r s(L)$ of the leftmost angular position of the canal with the radius $r \mathrm{~s}(L n)$ of the angular position without multiple intersections (as shown in Fig. 2(A)), meaning that the radius of an angular position in-between the two angular positions is linearly interpolated by $r s(L)$ and $r s(L n)$. Similarly, the right pedicle, lamina and transverse process can be excluded from the body by the boundary interpolated from the radius $r s(R)$ of the rightmost angular position of the canal with the radius $r s(R n)$ of the angular position without multiple intersections.

3. Determine the center of vertebral body by averaging the boundary pixels (already excluding the cracks, pedicle, lamina and transverse process) at the 360 angular positions (Fig. 2(B)), then repeat the above step to determine the radius of every angular position, e.g., the radii $(r(L)$ and $r(R))$ from the center to the leftmost and rightmost angular position of the canal. Then, we use the new 360 radii of the 360 boundary pixels to approximate a Bspline curve for the vertebral body [27].

4. Determine the middle point $C$ on the boundary of the canal. Its angular position to the center of the vertebral body is the average of the leftmost and rightmost angular positions of the concave feature enclosing the canal, and its radius can be calculated using the B-spline curve (as shown in Fig. 2(B)).

5. Determine the anteroposterior diameter $C D$ following the location of the opposite point $(D)$ of $C$. Use vectors starting from $C$ across the canal boundary (first intersection) to intersect other bone boundaries. A range of intersections with large distances are considered intersecting with the spinal process. The average vector of the leftmost and rightmost vectors of the range intersects $D$ (Fig. 2 (C)). If the spinal process is not resolved in the transverse section. Then, the spinal space is used to locate $D$. We use vectors starting from $C$ across the canal to intersect the air. A range of intersections that intersected the spinal space can be obtained (Fig. 2 (D)). Then, the average vector of the leftmost and rightmost vectors of the range intersects $D$ (Fig. 2 (D)). If this section resolves no spinal process and space, the vectors stating from $C$ to opposite boundary of the canal are approximated as a quadric radial function. The vector that is the local maximum of the function intersects $D$ (Fig. 2 (E)).

2.3 Compression Ratio Determination and
Recovering Angle and Distance Calculation from Transverse Sections

The anteroposterior diameter calculation method, described in the last section, determines the diameter on each section. Because the diameters of transverse sections in a normal vertebral bone should be near the same, the average of the diameters of all the transverse sections can represent the canal diameter in this vertebral bone. However, if some diameter has large deviation to the average, it is abandoned, and the average is re-determined. The normal (before fracture) diameter of the fractured vertebral bone is then, interpolated or extrapolated from neighboring bones of the fractured bone. The compression ratio on any transverse section of the fractured bone is the ratio of the anteroposterior diameter on the section to the interpolated or extrapolated normal value. The worst (smallest) ratio is used to represent as the compression ratio by the fracture.

We compute the angle and distracted or compressed distance for recovering using the centers calculated in all transverse sections as the following procedures.

1. Determine a centerline for each vertebral body that regress the centers of the transverse sections passing this body with least square errors [28]. The two ends of the line are located on the middles of the above and below neighboring disc spaces. However, if there exists some center with large deviation, the center is neglected and the line is re-determined (Fig. 3(A)). However, if the distance between the centers of two neighboring sections is too large, a shear dislocation is considered existing inside the fractured vertebral bone (Fig. 3(B)). Then, two respective centerlines are used to regress the two dislocated parts of the body. Then, the distance between the two lines is considered the shear dislocation by the fracture.

2. Determine the compressed angle by the centerlines of above and below neighboring vertebral bodies of the fractured body. $\alpha$ represents the angle of the two lines after the fracture (Fig. 3(D)) and $\beta$ represents the angle of the two lines if no fracture (Fig. 3(E)) that follows the normal anatomic (first or secondary) curve [29].

3. Determine the distracted or compressed distance. The length of a centerline represent the height of a vertebral body, while the normal height (before the fracture) of the fractured body can be interpolated or extrapolated by the lengths of neighboring vertebral bodies (without the fracture). Then, the distracted or compressed distance is the difference of the interpolated normal height with the height (length of the centerline) of the current fractured body. 


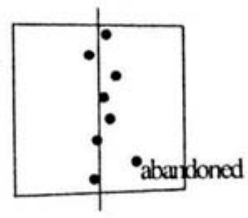

(A)

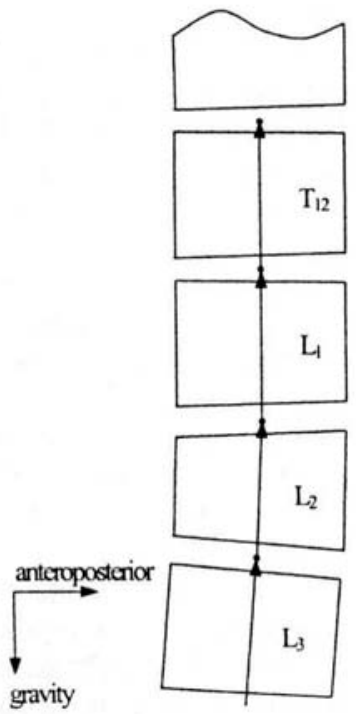

(C)

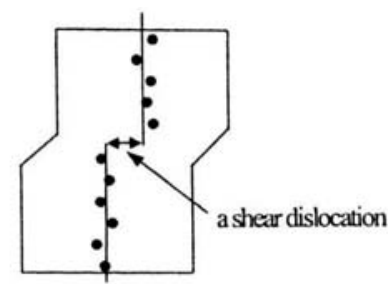

(B)

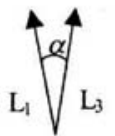

(D)

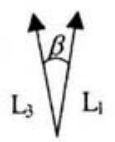

(E)

Fig.3 Determination of anatomic curve of a fractured spine

(A) Centerline of a vertebral body determined by regressing the centers on all sections passing the body

(B) Shear dislocation separated by two centerlines regressing two separate groups pf centers

(C) Centerlines of vertebral bodies representing the anatomic curve after the fracture

(D) Angle between the two centerlines of the above and below vertebral bodies of the fractured body.

(E) Normal angle of the above and below vertebral bodies, indicating the angle after recovery

\section{IMPLEMENTATION}

The prototype system employing the above method can be implemented on a PC platform (currently P-IV $1.4 \mathrm{G}$ is used.) in which a volume visualization software renders the isosurfaces reconstructed from the volume consisted by the CT section using the marching cube algorithm [30] and the 3D MAX graphics software [31] represents the recovering height and angle for the reduced vertebral bone. Any series of CT sections following the DICOM protocol [32] can be the source data to use our system for the diagnosis of the compression and burst fractures. In the following, a patient with a typical fractured spine treated at the Orthopedic Department of Taipei Medical University Hospital in July 2001 was used to demonstrate the results implemented by our system.

\subsection{Case Study}

The patient was a 39-year-old man and suffered from a falling-down from six-meter height. The following abnormalities were noted during physical examination: abdomen distension; numbness over both flank areas and positive finding of low back pain on lateral bending of the both legs; hypoesthesis below the L2-distribution of both thigh; weak plantar flexion on right big toe test (+); Laseque's sign (positive finding with $35^{\circ}$ elevation of the both lower legs); absence of ankle jerk; sensory loss in the L2 and L3 dermatome. Frontal and lateral X-rays were also performed and revealed the compression at $\mathrm{L} 2$. These findings were indicative of spinal cord lesion below L2. CT (General Electric high speed CT/i) was performed in 48 transverse sections ( $256 \times 256$ resolutions) from L3 to T11 with all 7 degrees oblique to the transversal plane and constant $3 \mathrm{~mm}$ intervals. These sections were taken to be perpendicular to L2. Fig. 4 shows a section 


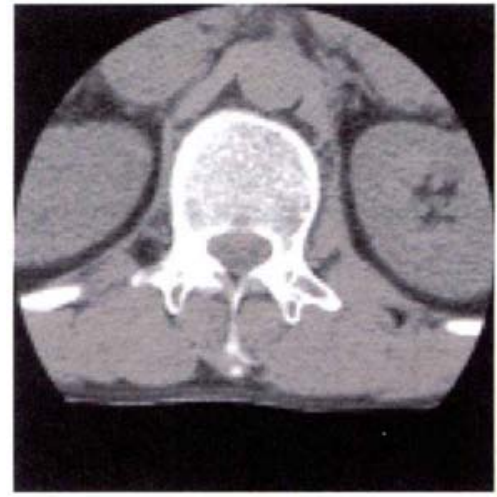

(A)

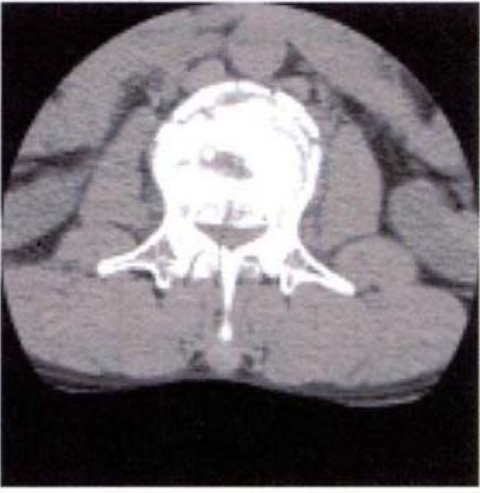

(B)

Fig. 4 CT transverse sections of vertebral bones (A)A section passes $\mathrm{L} 1$ without fracture (B)A section passes $\mathrm{L} 2$ with fracture
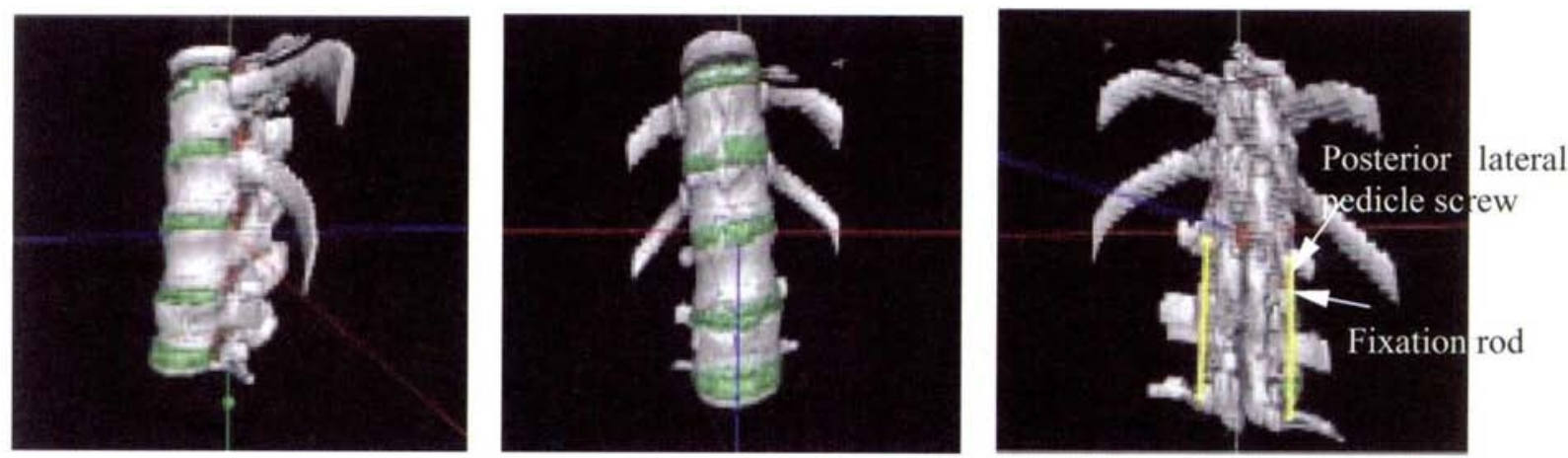

Fig. $53 \mathrm{D}$ image reconstructed from $48 \mathrm{CT}$ transverse sections (without interpolations)

$\triangle$ Gray area: bones. Green areas: disc spaces. Red areas: spinal code or roots. Yellow area: posterior lateral pedicle screws and reduction fixation rods

(A) Lateral view: indicates compression on $\mathrm{L2}$, especially the anterior column bringing kyphosis

(B) Frontal view: indicates un-symmetric horizontal compression on L2 bringing scoliosis

Posterior view: indicates positions for inserting posterior lateral pedicle screws and reduction rods for fracture reduction and posterior fusion

from $\mathrm{Ll}$ a normal vertebral bone demonstrating no canal compression and fracture, and a section from L2 demonstrating the canal compression and fracture. Fig. 5 shows 3D images that rendered the isosurfaces reconstructed from the marching cube algorithm. Fig. 5(A) shows the lateral view that demonstrates a serious compression on L2, especially the anterior column that brings kyphosis. Fig. 5(B) shows the frontal view that also demonstrates the un-symmetric horizontal compression on L2 that brings scoliosis. Fig. 5(C) shows the posterior view that demonstrates the positions for inserting posterior lateral pedicle screws for fracture reduction and posterior fusion.

Table 1 shows the computed canal diameter along the anteroposterior direction and the position of vertebral body center for each section. Table 2 shows the average diameter of the canal, height and centerline vector for each vertebral bone (T10, T11, T12, L1, L2 and L3). However, the fractured body L2 has a small shear dislocation, and such has two separate centerlines. This shear location is small, such cannot be observed from the 3D images as shown in Fig. 5. However, it is also a cause of bringing kyphosis and scoliosis. The assumed normal height and average canal diameter of the fractured body $\mathrm{L} 2$ are $33.1 \mathrm{~mm}$ and $11.8 \mathrm{~mm}$. The worst reduced ratio of the canal diameter is a severe $50 \%$. Fig. $6(\mathrm{~A})$ shows the images of the recognized (B-spline approximate) vertebral bodies wherein the pedicle, lamina, transverse and spinal process were excluded. Therefore, the bone fragment (with a crack as shown in Fig. 4(B)) burst into the canal at $L 2$ can be easily observed.

Fig. 6(B) shows a simplified 3D image revealing the relations between the vertebral bodies in which the thickness of a cylinder is the height of a vertebral body, the axis direction of the cylinder is the direction of the 
Tab. 1 Canal diameter along the anteroposterior direction and center $(x, y$, and $z$ coordinates) of vertebral body at each section

$\triangle$ T10: Section No.1-3, T11:7-12, T12:16-22, L1:26-33, L2:38-42, L3: 47-48.

Other sections are dise spaces but some are mixed with a space and a bone. Bold entries represent the $C D$ distances that have large deviation when determining average of the diameters and are abandoned.

\begin{tabular}{|c|c|c|c|c|}
\hline Section No. & $C D$ distance & $x$ coordinate & $v$ coordinate & $z$ coordinate \\
\hline 1 & 15.25 & -10.55 & 14.65 & 141.00 \\
\hline 2 & 12.89 & -10.55 & 15.23 & 138.00 \\
\hline 3 & 12.94 & -12.30 & 15.23 & 135.00 \\
\hline 4. & 13.53 & -12.30 & 16.41 & 132.00 \\
\hline 5 & 12.30 & -9.96 & 16.99 & 129.00 \\
\hline 6. & 11.78 & -10.55 & 15.82 & 126.00 \\
\hline 7 & 12.94 & -9.96 & 15.82 & 123.00 \\
\hline 8 & 13.49 & -9.96 & 15.82 & 120.00 \\
\hline 9 & 15.28 & -9.96 & 16.99 & 117.00 \\
\hline 10 & 14.65 & -8.79 & 14.06 & 114.00 \\
\hline 11 & 14.06 & -7.62 & 14.65 & 111.00 \\
\hline 12 & 12.30 & -8.79 & 15.23 & 108.00 \\
\hline 13 & 12.89 & -6.45 & 16.99 & 105.00 \\
\hline 14 & 13.48 & -8.20 & 17.58 & 102.00 \\
\hline 15 & 10.55 & -7.62 & 16.41 & 99.00 \\
\hline 16 & 11.78 & -7.62 & 16.99 & 96.00 \\
\hline 17 & 11.78 & -8.20 & 16.41 & 93.00 \\
\hline 18 & 12.94 & -5.27 & 17.58 & 90.00 \\
\hline 19 & 14.65 & -5.27 & 18.16 & 87.00 \\
\hline 20 & 11.72 & -4.69 & 17.58 & 84.00 \\
\hline 21 & 11.73 & -4.69 & 18.75 & 81.00 \\
\hline 22 & 11.19 & -4.10 & 19.34 & 78.00 \\
\hline 23 & 11.19 & -4.69 & 19.92 & 75.00 \\
\hline 24 & 11.73 & -4.69 & 19.92 & 72.00 \\
\hline 25 & 11.15 & -2.34 & 19.92 & 69.00 \\
\hline 26 & 10.56 & -8.20 & 22.27 & 66.00 \\
\hline 27 & 12.90 & -5.27 & 21.09 & 63.00 \\
\hline 28 & 15.99 & -4.69 & 22.27 & 60.00 \\
\hline 29 & 17.73 & 0.59 & 21.68 & 57.00 \\
\hline 30 & 13.01 & -1.76 & 22.27 & 54.00 \\
\hline 31 & 13.49 & -1.76 & 22.85 & 51.00 \\
\hline 32 & 13.01 & -1.76 & 24.02 & 48.00 \\
\hline 33 & 11.73 & -1.76 & 22.85 & 45.00 \\
\hline 34 & 11.73 & -3.52 & 25.20 & 42.00 \\
\hline 35 & 8.79 & 1.17 & 26.37 & 39.00 \\
\hline 36 & 8.81 & -0.59 & 25.20 & 36.00 \\
\hline 37 & 6.45 & -2.34 & 25.78 & 33.00 \\
\hline 38 & 5.86 & -2.34 & 25.78 & 30.00 \\
\hline 39 & 8.22 & -1.17 & 25.78 & 27.00 \\
\hline 40 & 7.03 & -1.17 & 25.78 & 24.00 \\
\hline 41 & 11.78 & 0.00 & 31.05 & 21.00 \\
\hline 42 & 11.13 & 0.59 & 30.47 & 18.00 \\
\hline 43 & 10.56 & 0.59 & 30.47 & 15.00 \\
\hline 44. & 11.73 & 0.59 & 35.74 & 12.00 \\
\hline 45 & 10.61 & -1.17 & 33.40 & 9.00 \\
\hline 46 & 11.95 & -1.17 & 33.40 & 6.00 \\
\hline 47 & 11.15 & 0.00 & 36.33 & 3.00 \\
\hline 48 & 11.19 & -0.59 & 33.98 & 0.00 \\
\hline
\end{tabular}

$\boldsymbol{x}$ : horizontal direction

$y$ : anteroposterior direction

$z:$ gravity direction

in $\mathbf{m m}$ 


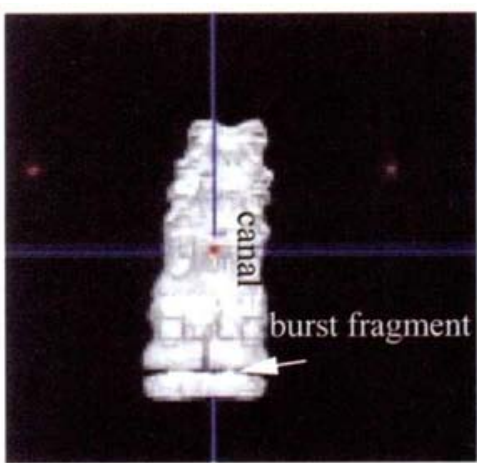

(A)

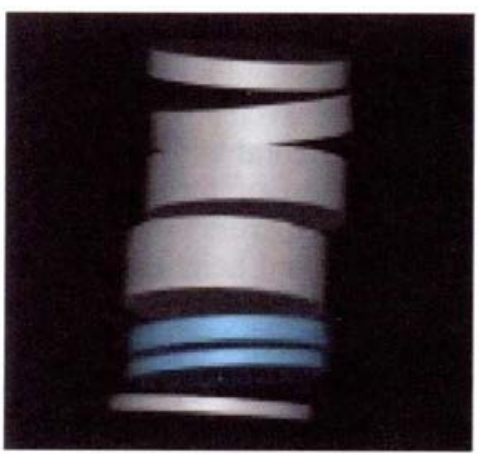

(D)

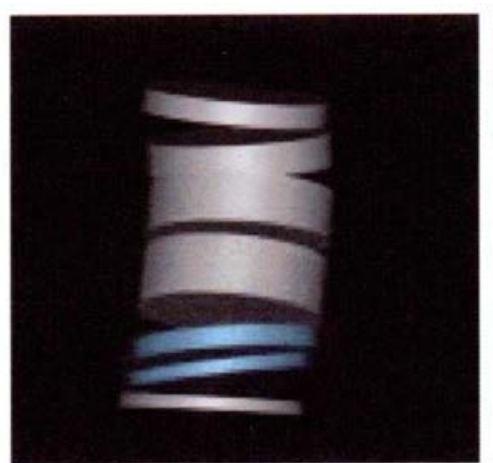

(B)

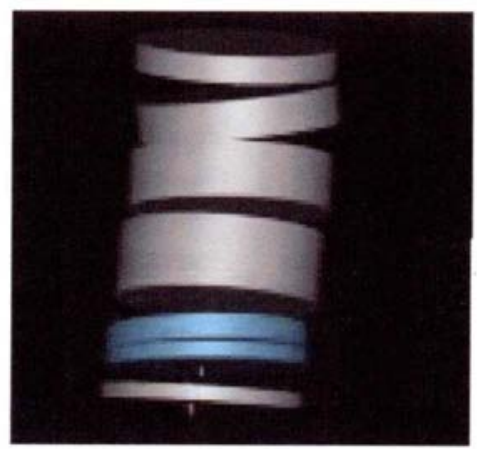

(E)

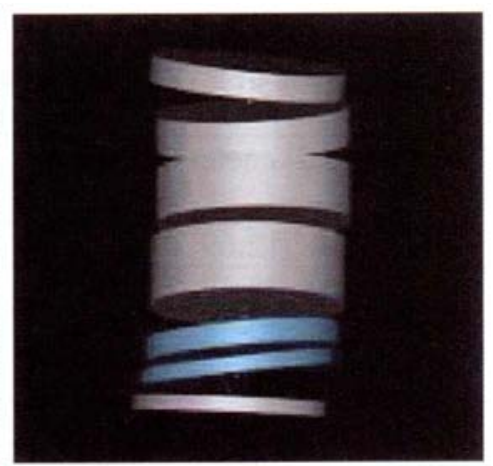

(C)

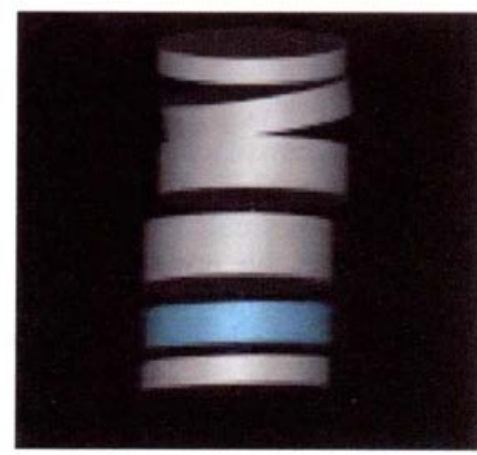

(F)

Fig.6 3D image abalysis for $48 \mathrm{CT}$ transverse sections

$\triangle$ Gray areas: bones. Green areas: disc spaces. Red areas: nerve code or roots. Blues areas: fractured bone, represented as two parts because the shear dislocation induces two different centerlines.

(A) Posterior view: indicates burst fragment to the canal at $L 2$, , only recognized vertebral bodies were reconstructed

(B) 3D simplified vertebral bodies with the fractured body $L 2$

(C) Interpolation of the heights and angles between the fractured $L 2$ and the assumed normal L2

(D) Interpolation of the heights and angles between the fractured $L 2$ and the assumed normal $L 2$

(E) Interpolation of the heights and angles between the fractured $L 2$ and the assumed normal $L 2$

(F) 3D simplified vertebral bodies with the assumed normal $L 2$ body wherein the height is interpolated by neighboring bodies and the angle inferred from the normal anatomic curve

centerline and the radius of the cylinder is the average radius of the B-spline approximate body. By comparing Fig. 6(B) with Fig. 5(A) wherein the body centerline directions and heights are similar, the centerlines of the bodies were correctly calculated can be observed. Fig. 6(F) shows a simplified 3D image in which the cylinder of L2 uses the assumed normal (before the fracture) centerline (direction and height) not the calculated fractures centerlines. Fig. 6(C), Fig. 6(D) and Fig. 6(E) show the results of morphing from Fig. 6(B) until Fig. 6(F).

The operation based on the calculated reduced angle and height was implemented. The above clinical syndrome disappears immediately after the surgery, and lateral and anteroposterior X-rays show good immediate reduction of bones. Lateral and anteroposterior X-rays of one year latter follow-up show good union of bones by the surgery.

\section{DISCUSSION}

In this study, we developed a diagnostic method that can automatic determine the compression ratio, the reduction angle and height for recovering the fractured bone. The 3D characteristic of the fractured bone, the bone fragment bursting into the canal, the relation between the fractured bone with neighboring bones and the spinal cord, and the angle and height for recovering can be clearly revealed. Using this tool, clinicians can quantitatively and accurately, with a spatial concept, evaluate conservative or surgical modalities and procedures for fractured bones.

A typical patient with the burst and compression fracture was used as the example, demonstrating that the compression and shear dislocation of the vertebral body, the burst fragment to the canal, and the reduced 
Tab. 2 Average diameter of canal, height and centerline normal vector of vertebral bone $L 2$ is represent as two parts because of a shear dislocation inside it.

\begin{tabular}{|c|c|c|c|}
\hline & A verage diameter of Canal & height & $\begin{array}{c}\text { Centerline Normal } \\
\text { Vector }(x, y, z)\end{array}$ \\
\hline $\mathrm{T} 10$ & 12.69 & **** & $(0.16, \quad 0.01, \quad 0.99)$ \\
\hline $\mathrm{T} 11$ & 13.49 & 15.12 & $(0.06, \quad-0.12, \quad 0.99)$ \\
\hline $\mathrm{T} 12$ & 12.34 & 21.46 & $(-0.15, \quad 0.15,0.98)$ \\
\hline $\mathrm{Ll}$ & 12.45 & 27.31 & $(-0.16, \quad 0.21, \quad 0.97)$ \\
\hline $\mathrm{L} 2 \mathrm{a}$ & 8.8 & 9.08 & $(-0.13,0.00,0.99)$ \\
\hline $\mathrm{L} 2 \mathrm{~b}$ & $* * *$ & 12.18 & $(-0.87,-0.05,0.49)$ \\
\hline L3 & 11.17 & $* * *$ & $(-0.10, \quad 0.10,0.99)$ \\
\hline
\end{tabular}

\author{
$X:$ horizontal direction \\ $Y$ : anteroposterior direc- \\ tion \\ $\boldsymbol{Z}$ : gravity direction
}

in $\mathbf{m m}$ height and angle can be accurately evaluated. The diagnostic results were compared with the intraoperative findings and were consistent at more than 1-year postoperative follow-up. This example indicate the surgical procedures uses the calculated reduced angle and height can recover the normal spinal anatomy, thus achieve anatomic stability, release the syndrome of nerve compression and bone pain and avoid future kyphosis and scoliosis.

Our method can represent realistic 3D images using our volume visualization software. Our volume visualization and surgical simulation software can render isosurfaces reconstructed from volumes and simulate musculoskeletal surgeries. However, because of the inheritance of volume structure, our method can only translate anatomic structures but cannot rotate structures. Therefore, we use commercial graphic software to simplify the recovering results by the decompression and fusion surgery. This is our future work that extends the surgical simulation software to simulate the decompression and fusion surgery so that realistic 3Dimages can be used to demonstrate the procedures and results of the decompression and fusion surgery.

\section{CONCLUSION}

The 3D geometry estimation of fractured bones and spinal anatomic curve, and the compression ration evaluation are important factors in deciding the appropriate diagnostic modality and treatment procedures for a spine fracture. Currently, fracture diagnosis using transverse sections is performed mainly based on clinician experience and without the benefit of qualitative and quantitative analyses. In this study, we proposed a method for computing the compression ratio and analyzing the 3D geometry of a fractured bone and neighboring bones to estimate the deformation of the anatomic curve so that precise surgical procedures for reduction and union on the spine can be evaluated.

Our method uses a B-spline curve to approximate the vertebral body boundary and a concave feature enclosing the canal on a transverse section, thus to determine the center of a vertebral body and the diameter and the compression ration of the canal. Then, our method uses centerlines to regress the centers of transversal sections. The deformed height and angle of the fractured bone can be estimated by the centerlines of the fractured bones and its neighboring bones, so that to determine the reduction height and angle for recovering the fracture. These estimations can be demonstrated in 3D images using any commercial CAD or graphics software.

Example of a patient with burst and compression bones demonstrated that the technique allows clinicians to quantitatively evaluate the compression ratio to the canal, the compressed height of the fractured body and the deformation of the anatomic curve. Combining this technique with 3D isosurface reconstruction and rendering techniques also facilitate automated surgical planning. In conclusion, our method appears to be feasible for automated fracture spine diagnosis and surgical management on CT transverse sections.

\section{ACKNOWLEDGMENT}

This study was partially sponsored by the $\mathrm{Na}$ tional Science Council (NSC), Taiwan/ROC; grant numbers NSC-91-2213-E-033-024 and NSC-91-2314B-038-034.

\section{REFERENCE}

1. Hu RW: Orthopaedic Knowledge Update, Trauma 2. In: Kellam JF, Fischer TJ, Tornetta III P, Bosse MJ, Harris MB, editors. Evaluation and assessment of the polytrauma patient for spinal injuries, Rosemont: American Academy of Orthopaedic Sur- 
geons $2000 ; 319-328$.

2. An HS, Andreshak TG, Nguyen C, Williams A and Daniels D: Can we distinguish between benign versus malignant compression fractures of the spine by magnetic resonance imaging. Spine 1995; 20: 1776-1782.

3. Van Beek EJR, Been HD, Ponsen KJ and Mass M: Upper thoracic spinal fractures in trauma patients a diagnostic pitfall. Injury, Int. J. Care Injured 2000; 31: 219-223.

4. James BH: Orthopaedic knowledge update. In: Howard S, Randall TL, (Eds), Spine, Rosemont, American Academy of Orthopaedic Surgeons 1999; 561-754.

5. Garfin SR and Vaccaro AR: Orthopaedic knowledge update. In: Garfin SR, Vaccaro AR, (Eds), Spine, Rosemont, American Academy of Orthopaedic Surgeons 1997; 55-61.

6. Regan JJ, Yuan H and McAfee PC: Laparoscopic fusion of the lumbar spine: minimally invasive spine surgery. A prospective multicenter study evaluating open and laparoscopic lumbar fusion. Spine 1999; 24-4: 402-411.

7. Chao EY, Barrance P, Genda E, Iwasaki N, Kato S and Faust A: Virtual reality (VR) techniques in orthopaedic research and practice. Stud Health Technol Inform 1997; 39:107-114.

8. Wood KB: Orthopaedic knowledge update, Trauma 2. In: Kellam JF, Fischer TJ, Tornetta III P, Bosse MJ, Harris MB, editors. Thoracic spine injuries, Rosemont: American Academy of Orthopaedic Surgeons 2000; 371-381.

9. Hsieh MS and Tsai MD: Diagnosis of herniated intervertebral disc assisted by three-dimensional, multiaxial, magnetic resonance imaging. J Formos Med Assoc 1999; 98-5: 347-355.

10. Greenspan A: Orthopedic radiology, A Practical approach $3^{\text {rd }}$ ed. LW and W, USA $1994 ; 371-390$.

11.Denis $F$ : The three column spine and its significance in the classification of acute thoracolumbar spinal injuries. Spine 1983; 8: 817-831.

12. Gertzbin SD: Spine update: Classification of thoracic and lumbar fractures. Spine 1994; 19: 626628.

13. Magerl F, Aebi M, Gertzbein SD, Harms $J$ and Nazarian S: A comprehensive classification of thoracic and lumbar injuries Eur J Spine 1994; 3: 184201.

14. Daniaux H, Seykora $P$, Genelin A, Lang $T$ and Kathrein A: Application of posterior plating and modifications in thoracolumbar spine injuries. Indication, techniques, and results. Spine 1991; 16: 125-133.

15.Daniaux $\mathrm{H}$ : Transpedicular repositioning and spongiosaplasty in fractures of the vertebral bodies of the lower thoracic and lumbar spine. Unfallchirurg 1986; 89: 197-213.
16. Esses SI, Botsford DJ, Wright T, Bednar D and Bailey $S$ : Operative treatment of spinal fractures with the AO internal fixator. Spine 1991; 16: 146150.

17. Karlstrom G, Olerud S and Sjostrom L: Transpedicular fixation of thoracolumbar fractures. Contemp Orthop 1990; 20: 285-300.

18. Danisa OA, Shaffrey CI, Jance JA, Whitehill R, Wang GJ and Szabo TA: Surgical approaches for the correction of unstable thoracolumbar burst fractures: a retrospective analysis of treatment outcomes. J Neurosurg 1995; 83: 977-983.

19. Hitchon PW, Tomer JC, Haddad SF and Follett KA: Management options in thoracolumbar burst fractures. Surg Neurol 1998; 49: 619-626.

20. Andress HJ, Braun H, Helmberger T, Schürmann, Hertlein $\mathrm{H}$ and Hartl WH: Long-term results after posterior fixation of thoraco-lumbar burst fractures. Injury, Int. J. Care Injured 2002; 33: 357-365.

21. Theodorou DJ, Theodorou SJ, Duncan TD, Garfin SR and Wong WH: Percutaneous balloon kyphoplasty for the correction of spinal deformity in painful vertebral body compression fractures. Clinical Imaging 2002; 26: 1-5.

22. Been HD and Bouma GJ: Comparison of two Types of Surgery for Thoraco-Lumbar Burst Fractures: Combined Anterior and Posterior Stabilisation vs. Posterior Instrumentation Only. Acta Neurochirurgica $1999 ; 141: 349-357$.

23. Knop C, Fabian HF, Bastian L and Blauth M: Late Results of thoracolumbar Fractures After Posterior Instrumentation and Transpedicular Bone Grafting. Spine 2001; 26-1: 88-99.

24. Panda B and Chatteji BN: Least square generalized B-spline signal and image processing. Signal Processing 2001; 81: 2005-2017.

25. Chuang SHF and Kuo CZ: One-sided arc approximation of $\mathbf{B}$-spline curves for interference-free offsetting. Computer-Aided Design 1999; 31 : 111 -

26. T88i MD, Hsieh MS and Jou SB: A New Method for Lumbar Herniated Inter-Vertebral Disc Diagnosis based on Image Analysis of Transverse Sections. Comput Med Imag Grap. Page proof completed. Otherwise, the following relating conference paper can be referred.

27. Tsai MD, Shih JY and Hsieh MS: An Image Analysis Method for Inter-Vertebral Disc Sections with Application in Diagnosis of Lumbar Disc Herniation. Proceedings of CVGIP2002, 2002: appear in 25, Aug.

28. Burger $P$ and Gillies, D: In: Interactive Computer Graphics, Cubic B-spline. Addison Wesley company, New York 1989; 250-264.

29. Cheney $W$ and Kincaid D: Smoothing of data and the method of least squares. In: Numerical Mathematics and Computing, Brooks/Cole(Eds.), 1994; $381-409$. 
30.Bridwell and DeWald: The textbook of spinal surgery. $2^{\text {nd }}$ ed. Philadelphia: JB Lippincott 1996; 570.

31.Lorensen WE and Cline HE: Marching Cubes: A High Resolution 3D Surface Construction Algorithm. ACM SIGGraph Computer Graphics. 1987; Addision Wesley press: 1987; 163-169.

32.Boardman T: 3ds max 4fundamentals. New Riders Publishing press: Dwyer D, 2001.

33. Bernarding J, Thiel I, Decker l. and Tolxdorff T: Implementation of a dynamic platform- independent DICOM-server. Computer Methods and Programs in Biomedicine. 2001; 65: 71-78.
34. Tsai MD, Jou SB and Hsieh MS: Accurate Surface Voxelization for Manipulating Volumetric Surfaces and Solids with Application in Simulating Musculoskeletal Surgery. Pacific Graphics. 2001; IEEE CS press: 2001; 234-243.

35. Tsai MD, Hsieh MS and Jou SB: Virtual Reality Orthopedic Surgery Simulator. Comput Biol Med. 2001; 31(3): 333-351.

36. Hsieh MS, Tsai MD and Chung WC: Virtual Reality Simulator for Osteotomy and Fusion Involving the Musculoskeletal System, Comput Med Imag Grap. 2002; 26(2): 91-101. 